\title{
Use of Gliricidia sepium as a Nurse Plant to Reforest Man-made Grasslands in the Knuckles Forest Reserve, Sri Lanka
}

\author{
Gunasekara R.D.A. ${ }^{*}$, Gunaratne A. M. T. A. ${ }^{2}$, Senevirathne G. ${ }^{3}$, \\ Gunatilleke I. A. U. N. ${ }^{2}$ and Gunatilleke C. V.S. ${ }^{2}$ \\ ${ }^{l}$ Post Graduate Institute of Science, University of Peradeniya, Peradeniya, Sri Lanka \\ ${ }^{2}$ Department of Botany, Faculty of Science, University of Peradeniya, Peradeniya, Sri Lanka \\ ${ }^{3}$ Institute of Fundamental Science, Kandy, Sri Lanka \\ *rdaguna@yahoo.com
}

\begin{abstract}
Biodiversity richness and socially beneficial watershed services are high in the Knuckles Forest Reserve. However, the lower montane forest patches on the eastern slopes of the KFR are highly fragmented mainly due to anthropogenic disturbances. If these forest fragments are not connected in the near future, they will disappear from the landscape due to their low regeneration rates. Native tree species when used for restoration of these lands faces many difficulties due to various biotic and abiotic stresses including harsh microclimatic conditions and infertile soils. Therefore, usage of a nurse plant will facilitate the growth of target species due to creation of favourable microclimatic conditions. Gliricidia sepium has been used as a shade plant in Sri Lanka, since colonial times. More recently it has been used for indigenous farming practices, soil stabilization, living fences and as fuel wood, animal forage, green manure. In our research we investigate the potential of $G$. sepium as a nurse plant to establish four native tree species on man-made grasslands in central Sri Lanka. The study was conducted on four blocks of grasslands. Four replicates of each of the three island sizes (small $4 \mathrm{~m}^{2}$, medium $16 \mathrm{~m}^{2}$, and large $64 \mathrm{~m}^{2}$ ) were created inside each block. One set of islands in each block were planted with $G$. sepium stakes at $2 \mathrm{~m}$ intervals in a grid format. The other set of islands in each block was kept as a control without planting G. sepium stakes. Seedlings of Macaranga indica, Bhesa ceylanica, Symplocos cochinchinensis and Eugenia bracteata were planted randomly in islands with and without $G$. sepium stakes.
\end{abstract}

After three months of establishment, the survival rate of $G$. sepium in the four blocks was more than $80 \%$ and the survival rate of the G. sepium was $20 \%$ higher in the small and medium islands than the large islands. The mean number of sprouts per stake was more than eight in medium and large islands and less than six in small islands. The mean survival rate was higher $(5.8 \%)$ and the mean growth rates was lower $(0.006 \%)$ for all the four native species with $G$. sepium than without $G$. sepium after three months of planting. G. sepium increased the survival rates of native species after three months of establishment due to favourable microclimatic conditions created by them. However, growth rate of the native species which were planted with $G$. sepium was lower probably due to interspecific competition. Therefore, G. sepium can be used as an effective nurse plant in initial phases of reforestation programmes to increase the survival rates of native tree species on degraded submontane sites in Sri Lanka.

Keywords: Reforestation, Man-made grasslands, Nurse plants, Native species 